\title{
Evaluation of Morphological Texture Features for Mangrove Forest Mapping and Species Discrimination Using Multispectral IKONOS Imagery
}

\author{
Xin Huang, Liangpei Zhang, and Le Wang
}

\begin{abstract}
This letter aims to exploit morphological textures in discriminating three mangrove species and surrounding environment with multispectral IKONOS imagery in a study area on the Caribbean coast of Panama. Morphological texture features are utilized to distinguish red (Rhizophora mangle), white (Laguncularia racemosa), and black (Avicennia germinans) mangroves and rainforest regions. Meanwhile, two fusion methods are presented, i.e., vector stacking and support vector machine (SVM) output fusion, for integrating the hybrid spectral-textural features. For comparison purposes, the object-based analysis and the gray-level co-occurrence matrix (GLCM) textures are adopted. Results revealed that the morphological feature opening by reconstruction (OBR) followed by closing by reconstruction (CBR) and its dual operator CBR followed by OBR gave very promising accuracies for both mangrove discrimination $\mathbf{8 9 . 1 \%}$ and $91.1 \%$, respectively) and forest mapping $(91.4 \%$ and $93.7 \%$, respectively), compared with the object-based analysis $(80.5 \%$ for mangrove discrimination and $82.9 \%$ for forest mapping) and the GLCM method $(81.9 \%$ and $87.2 \%$, respectively). With respect to the spectral-textural information fusion algorithms, experiments showed that the SVM output fusion could obtain an additional $\mathbf{2 . 0 \%}$ accuracy improvement than the vector-stacking approach.
\end{abstract}

Index Terms-Mangrove, mathematical morphology, objectbased analysis, texture.

\section{INTRODUCTION}

$\mathbf{M}$ ANGROVES are a unique forest type, located at the confluence of the land-sea interface along low-energy coastlines and estuaries throughout the tropics and in many subtropical areas. Mangrove forests sustain both ecologically and economically important ecosystems. However, their health and persistence are gravely threatened by various forms of coastal developments [1]. Therefore, there is an increasing need to monitor mangrove forest structure and dynamics on a regular basis so that effective management can be achieved. To this end,

Manuscript received November 19, 2008; revised December 22, 2008 and January 16, 2009. First published February 24, 2009; current version published July 4,2009 . This work was supported in part by the 863 High Technology Program of China under Grant 2007AA12Z148 and in part by the National Science Foundation of China under Grant 40771139.

X. Huang and L. Zhang are with the State Key Laboratory of Information Engineering in Surveying, Mapping, and Remote Sensing, Wuhan University, Wuhan 430079, China (e-mail: huang_whu@163.com; zlp62@public.wh. hb.cn).

L. Wang is with the Department of Geography, The State University of New York at Buffalo, Buffalo, NY 14261 USA (e-mail: lewang @ buffalo.edu).

Color versions of one or more of the figures in this paper are available online at http://ieeexplore.ieee.org.

Digital Object Identifier 10.1109/LGRS.2009.2014398 remote sensing constitutes a cost-effective avenue to assist in this effort.

Some studies have been reported to exploit remote-sensing data for mangrove mapping. Gao [2] developed a twotiered method using multispectral SPOT imagery to map the mangroves in New Zealand. The results showed that mangroves could only be mapped more accurately if they were classified as two separate classes and merged together after the classification. In another study, the relative importance of spatial and spectral resolutions in mangrove mapping was compared [3]. The results revealed that a high spectral resolution [Thematic Mapper (TM)] was more important in accurately mapping mangroves than a fine spatial resolution (SPOT). Green et al. [4] found that accurate discrimination among mangrove species was not possible using Landsat TM or SPOT XS data but was feasible using Compact Airborne Spectrographic Imager airborne data. Recently, with the availability of very high resolution (VHR) images, accurate mapping for mangrove species becomes possible since rich spatial features can be exploited. For the first time, Wang et al. [1] demonstrated that spectral information can be used to effectively discriminate different species of mangroves in high-resolution imagery, suggesting that analyses of broadband multispectral imagery is a promising tool for mangrove conservation. A recent study from Wang et al. [14] revealed that a clustering-based neuralnetwork method can better accommodate second-order textural information in mangrove species classification.

In this context, this letter aims to exploit the spatial/textural features for mangrove species discrimination using multispectral IKONOS imagery. The novel aspects of this letter are as follows: 1) The dual operators opening by reconstruction (OBR) followed by closing by reconstruction (CBR) (OFC) and CBR followed by OBR (CFO) are proposed to extract textural features of mangrove forests; 2) the morphological profiles of the two operators are defined as multiscale texture representations; and 3) a support vector machine (SVM) output fusion approach is presented to integrate the multispectral and textural features.

\section{Morphological TeXture EXtraction}

Mathematical morphology is an effective tool for extracting image components that are useful in the representation and description of region features. Morphological features are based on some basic operators, such as opening and closing, used 
to remove small bright (opening) or dark (closing) details while leaving the overall features relatively undisturbed. The morphological reconstruction is another commonly used technique that has been proved to have better shape preservation than classical morphological operators [5], [13]. Morphological features have been successfully applied to urban VHR images [5], [11]. In particular, some operations, such as gradients, top hats, and opening/closing by reconstruction, have been proved to be efficient for texture extraction and segmentation of natural landscapes [12]. However, few studies have been reported where morphological features have been used for mangrove species discrimination. In addition, in this letter, several new operators, such as OFC and its dual transformation CFO, are presented for texture analysis, and they are also extended to multiscale profiles using a series of sizes of structural elements (SEs). These new transformations are defined based on some commonly used operators.

1) Erosion and dilation of $f$ by SE: $\varepsilon^{\mathrm{SE}}(f)$ and $\delta^{\mathrm{SE}}(f)$.

2) Opening and Closing of $f$ by SE

$$
\gamma^{\mathrm{SE}}(f)=\delta^{\mathrm{SE}}\left(\varepsilon^{\mathrm{SE}}(f)\right) \text { and } \phi^{\mathrm{SE}}(f)=\varepsilon^{\mathrm{SE}}\left(\delta^{\mathrm{SE}}(f)\right) .
$$

3) Reconstruction of images $g$ and $f$ by $\mathrm{SE}: \operatorname{Rec}^{\mathrm{SE}}(g, f)$, where $g$, the marker, is the starting point for the transformation and $f$, the mask, constrains the transformation. The reconstruction aims to preserve the shapes of features since some morphological operators deform the objects. In this framework, the OBR and CBR can be expressed as

$$
\begin{aligned}
& \gamma_{\mathrm{R}}^{\mathrm{SE}}(f)=\operatorname{Rec}^{\mathrm{SE}}\left(\varepsilon^{\mathrm{SE}}(f), f\right) \\
& \phi_{\mathrm{R}}^{\mathrm{SE}}(f)=\operatorname{Rec}^{\mathrm{SE}}\left(\delta^{\mathrm{SE}}(f), f\right) .
\end{aligned}
$$

Afterward, OFC and CFO, as well as their multiscale extensions, can be defined as follows.

1) OFC, which is an improved version of OBR [6]. It aims to clean up the OBR image and remove some small noise in homogeneous regions. OFC is defined as

$$
\mathrm{OFC}^{\mathrm{SE}}(f)=\phi_{\mathrm{R}}^{\mathrm{SE}}\left(\gamma_{\mathrm{R}}^{\mathrm{SE}}(f)\right) .
$$

2) $\mathrm{CFO}$, which is an improved version of CBR and is a dual transformation of OFC. It is computed by implementing $\mathrm{CBR}$ and OBR operations in turn

$$
\mathrm{CFO}^{\mathrm{SE}}(f)=\gamma_{\mathrm{R}}^{\mathrm{SE}}\left(\phi_{\mathrm{R}}^{\mathrm{SE}}(f)\right) .
$$

3) Morphological profiles of OFC and CFO, which are multiscale representations of the two operators and are obtained using a series of SE sizes [13]. Define $\prod_{\text {OFC }}(x)$ and $\prod_{\mathrm{CFO}}(x)$ as the profiles of OFC and CFO, respectively, at the pixel $x$ of image $f$

$$
\begin{aligned}
& \prod_{\mathrm{OFC}}(x)=\left\{\prod_{\mathrm{OFC}}^{\mathrm{SE}}: \prod_{\mathrm{OFC}}^{\mathrm{SE}}(x)=\mathrm{OFC}^{\mathrm{SE}}(x) \quad \forall \mathrm{SE} \in[0, n]\right\} \\
& \prod_{\mathrm{CFO}}(x)=\left\{\prod_{\mathrm{CFO}}^{\mathrm{SE}}: \prod_{\mathrm{CFO}}^{\mathrm{SE}}(x)=\mathrm{CFO}^{\mathrm{SE}}(x) \quad \forall \mathrm{SE} \in[0, n]\right\}
\end{aligned}
$$

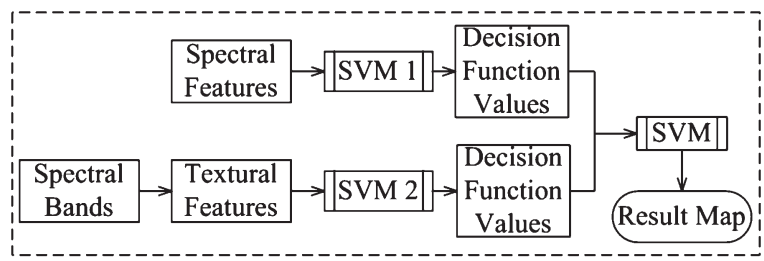

Fig. 1. Flowchart of SVM output fusion algorithm.

where $n$ is equal to the total number of iterations. In addition, the OFC and CFO features can be stacked together to extract both light and dark structures of the scene. The joint operator is written as $[\mathrm{CFO}+$ $\mathrm{OFC}^{\mathrm{SE}}(f)=\left\{\mathrm{CFO}^{\mathrm{SE}}(f), \mathrm{OFC}^{\mathrm{SE}}(f)\right\}$. Similarly, the multiscale profile of $[\mathrm{OFC}+\mathrm{CFO}]$ is defined by concatenating $\prod_{\mathrm{OFC}}(x)$ and $\prod_{\mathrm{CFO}}(x)$

$$
\prod_{\mathrm{OFC}+\mathrm{CFO}}=\left\{\prod_{\mathrm{OFC}}, \prod_{\mathrm{CFO}}\right\}
$$

Some commonly used morphological textures are also utilized for comparison, such as morphological gradient (MG), which is a measure of local variation and defined as $\mathrm{MG}^{\mathrm{SE}}(f)=\delta^{\mathrm{SE}}(f)-\varepsilon^{\mathrm{SE}}(f)$, and top hat by reconstruction (THR), which is used to extract light structures from images and is implemented by subtracting the OBR from the original image $\operatorname{THR}^{\mathrm{SE}}(f)=f-\mathrm{OBR}^{\mathrm{SE}}(f)$.

\section{Spectral-TeXtural Information Fusion}

Due to the similar spectral response between different mangrove species and between mangroves and other vegetations, it is necessary to integrate spectral and textural information for accurately mapping. A straightforward approach is to integrate the hybrid features in a single classifier, namely, the vector stacking. In this letter, SVM is used as the classifier considering its capability and robustness for complex classification problems. SVM is a fast and efficient machine learning algorithm and is insensitive to the prior distribution assumption. It has been successfully applied to multisensor information fusion [7] and object-based classification [10]. Therefore, it is worthwhile evaluating its ability for spectral-textural information fusion and mangrove discrimination.

Apart from the vector-stacking approach, the hybrid information can also be merged using SVM output functions. The spectral and textural features are processed separately in two SVMs, and the decision function values of the two SVMs are then integrated (Fig. 1). In this model, the discriminant function values output by SVMs represent the distance between the sample points and the decision hyperplane. The outputs of SVM1 (spectral SVM) and SVM2 (textural SVM) can be regarded as a preclassification. By applying the multiclass SVM, the spectral or textural features are transformed into a new feature space that is made up of the distance values for each class. The preclassification images are better comparable than the original features and are more appropriate to describe the interclass differences. This model was proposed by Waske and Benediktsson [7] for integrating optical and synthetic aperture radar data, while, in this letter, it is developed to fuse spectral 


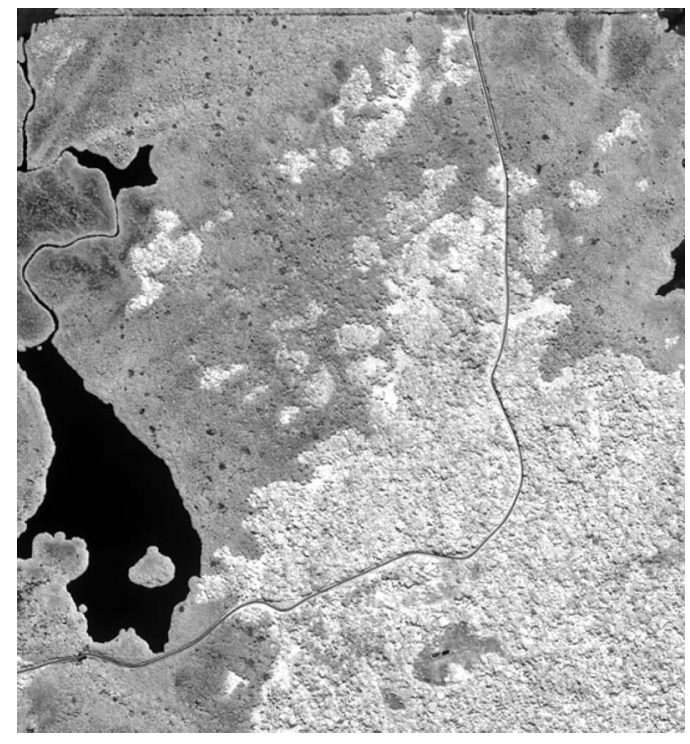

Fig. 2. Infrared channel of the study area.

and textural information for mangrove mapping due to its efficiency for multisource information processing.

\section{EXPERIMENTS AND DISCUSSION}

\section{A. Study Area and Data}

The study was conducted in mainland mangrove forests near the Smithsonian Tropical Research Institute's Galeta Marine Laboratory $\left(9^{\circ} 24^{\prime} 18^{\prime \prime} \mathrm{N}, 79^{\circ} 51^{\prime} 48.5^{\prime \prime} \mathrm{W}\right)$ at Punta Galeta on the Caribbean coast of Panama. There are three tree species in the study forests: black (Avicennia germinans), white (Laguncularia racemosa), and red (Rhizophora mangle) mangroves. Red mangrove forms a pure or nearly pure stand at the seaward fringe. About 10-20 m from the water's edge, white mangrove joins the canopy, forming a nearly even mixture with red mangrove in the low intertidal. In these mixed-species stands, white mangroves reach average heights of $22 \mathrm{~m}$, while red mangroves average 16-18 $\mathrm{m}$ in height. Black mangrove joins the canopy in the midintertidal, creating a mixed canopy of the three species, and gradually monopolizes most upper intertidal stands.

A cloud-free IKONOS image, at 4-m resolution, was acquired on June 13, 2000, at 3:24 pm local time. The infrared channel of the study area is shown in Fig. 2. Based on the field survey and investigation, the samples of seven land-cover types were chosen: red mangrove (44 samples for training and 700 for validation), white mangrove (46 and 446), black mangrove (48 and 369), gap (46 and 626), lagoon (43 and 618), $\operatorname{road}(42$ and 257), and rainforest (52 and 706). Table I listed the Jeffries-Matusita (JM) distance [8] of different features. It can be seen that the usage of OFC and CFO textures significantly enhanced the species discrimination ability.

\section{B. Comparative Algorithms in the Experiments}

The gray-level co-occurrence matrix (GLCM) textures and the object-based analysis are adopted for comparison purposes. The first- (variance) and second-order (contrast, correlation,
TABLE I

JM SEPARABILITy TEST FOR DifFERENT FEATURES $(\mathrm{R}=\mathrm{Red}$, $\mathrm{B}=$ Black, $\mathrm{W}=$ White, AND RF $=$ Rainforest)

\begin{tabular}{|c|c|c|c|c|c|c|}
\hline & R-RF & B-RF & R-B & W-B & W-RF & R-W \\
\hline \hline MS & 0.57 & 0.99 & 1.00 & 1.87 & 1.88 & 1.88 \\
\hline MS+PC1 & 0.58 & 1.00 & 1.00 & 1.87 & 1.88 & 1.88 \\
\hline MS+NDVI & 0.69 & 1.59 & 1.52 & 1.93 & 1.92 & 1.90 \\
\hline \hline THR & 1.56 & 1.35 & 1.19 & 1.90 & 1.95 & 1.92 \\
\hline MG & 1.23 & 1.16 & 1.22 & 1.89 & 1.89 & 1.90 \\
\hline OBR & 1.11 & 1.40 & 1.42 & 1.92 & 1.93 & 1.92 \\
\hline OFC & 1.99 & 1.98 & 1.60 & 1.93 & 1.99 & 1.97 \\
\hline CFO & 1.85 & 1.99 & 1.67 & 1.93 & 1.99 & 1.97 \\
\hline OFC+CFO & 1.99 & 1.99 & 1.79 & 1.93 & 1.99 & 1.98 \\
\hline
\end{tabular}

TABLE II

ACCURACIES For DifFERENT MORPHOLOGICAL TEXTURES

\begin{tabular}{|c|c|c|c|c|}
\hline \multirow{2}{*}{ Features } & \multicolumn{2}{|c|}{ Mapping } & \multicolumn{2}{c|}{ Discrimination } \\
\cline { 2 - 5 } & OA (\%) & Kappa & OA (\%) & Kappa \\
\hline \hline MS & 75.4 & 0.711 & 69.3 & 0.585 \\
\hline MS+PCA & 75.1 & 0.707 & 69.1 & 0.583 \\
\hline MS+NDVI & 75.5 & 0.712 & 69.2 & 0.584 \\
\hline \hline MG & 80.4 & 0.769 & 73.2 & 0.642 \\
\hline OBR & 84.6 & 0.819 & 81.6 & 0.752 \\
\hline CBR & 80.6 & 0.771 & 72.2 & 0.624 \\
\hline THR & 82.6 & 0.800 & 79.7 & 0.725 \\
\hline OFC & 91.4 & 0.900 & 89.1 & 0.852 \\
\hline CFO & 93.6 & 0.924 & 91.8 & 0.888 \\
\hline OFC+CFO & 93.5 & 0.923 & 91.2 & 0.882 \\
\hline
\end{tabular}

and entropy) statistics of the GLCM are calculated, and the fractal net evolution approach [9] is employed for the object-based analysis. In addition, a multilevel object-based technique [10] is also used to adaptively model the spatial context of each pixel.

\section{Results and Analysis}

In experiments, all the feature sets are classified using SVM with the radial basis function due to its effectiveness in many classification problems [10]. The accuracies are assessed using two test sets; all the test samples are used for validation of mangrove forest mapping, and a subset is chosen for assessment of mangrove species discrimination, including red, white, and black mangroves and rainforest.

1) Comparison of Different Morphological Features: The morphological textures are computed based on the normalized difference vegetation index (NDVI), and each of them is integrated with the four multispectral channels using the vectorstacking approach. The accuracies of both forest mapping and species discrimination are reported in Table II, from which it can be seen that all morphological textures substantially improved the spectral mapping. The OFC and its dual operator $\mathrm{CFO}$ gave very promising results. Comparing OFC with the spectral-only classification, the improvements of overall accuracy are $16.0 \%$ and $19.8 \%$ for forest mapping and species discrimination, respectively. The improvements achieved by CFO are $18.2 \%$ and $22.5 \%$, respectively. OFC (or CFO) performs satisfactorily because it is an improved version of OBR (or CBR) and it can reduce the spectral variation and noise in homogeneous regions and, at the same time, preserve discriminative information.

2) Multiscale Analysis: The OFC and CFO are analyzed with different sizes of SE based on the NDVI image. Results are 
(a)

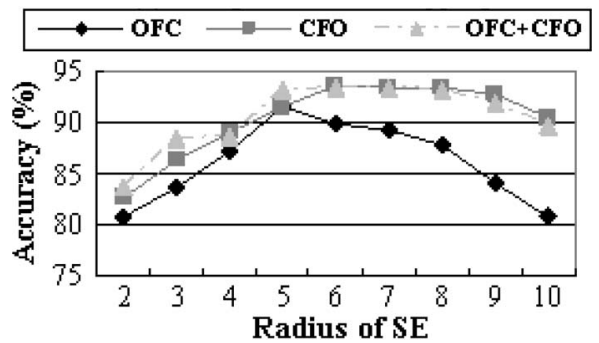

(b)

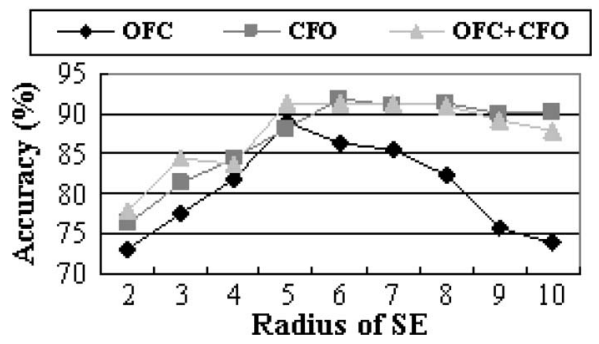

Fig. 3. Sensitivity analysis for $\mathrm{OFC}, \mathrm{OFC}$, and $[\mathrm{OFC}+\mathrm{CFO}]$.

TABLE III

Results of THE MORPHOLOGICAL PROFILES

\begin{tabular}{|c|c|c|c|c|}
\hline \multirow{2}{*}{ Profiles } & \multicolumn{2}{|c|}{ Mapping } & \multicolumn{2}{c|}{ Discrimination } \\
\cline { 2 - 5 } & OA (\%) & Kappa & OA (\%) & Kappa \\
\hline \hline OFC & 92.3 & 0.909 & 89.8 & 0.862 \\
\hline CFO & 93.7 & 0.925 & 91.1 & 0.879 \\
\hline OFC+CFO & 92.1 & 0.907 & 89.3 & 0.855 \\
\hline
\end{tabular}

shown in Fig. 3, where the horizontal axis represents the radius of the disk-shaped SE. It is seen that the $\mathrm{CFO}$ and $[\mathrm{OFC}+\mathrm{CFO}]$ operators obtained satisfactory accuracies with a radius ranging from five to ten (at least $90.5 \%$ for forest mapping and $87.8 \%$ for species discrimination). The joint operator $[\mathrm{OFC}+\mathrm{CFO}]$ outperformed CFO for small SEs (radius $=2,3,4$ ), while they gave very similar results when the radius is larger than four. It is worth noting that the OFC did not obtain high accuracies with radius $\geq 6$. This is because the OFC operator with a large radius can remove some bright features which may be informative for the mangrove forest in the NDVI image. The results of morphological profiles (OFC, $\mathrm{CFO}$, and the joint operator) were provided in Table III. The discrete sizes of SE for the profiles were defined with $\mathrm{SE} \in[0,7][(3)-(5)]$. The results revealed that the multiscale profiles could give comparable results with the highest accuracies obtained by the sensitivity analysis.

3) Information Fusion: Different spectral-textural models are shown in Fig. 4. The textural features are extracted by computing the CFO operator on four spectral bands. Fig. 4 showed that both fusion approaches effectively integrated the spectral-textural information and outperformed the spectral mapping. It is worth noting that SVM output fusion gave comparable or higher accuracies than the vector stacking for all the classes. The improvements achieved by the SVM fusion are $1.6 \%$ and $2.1 \%$ for forest mapping and species discrimination, respectively. However, it should be remembered that these improvements were obtained by additional SVMs.

4) Comparison Between the Morphological Texture, GLCM, and Object-Based Analysis: GLCM was computed based on

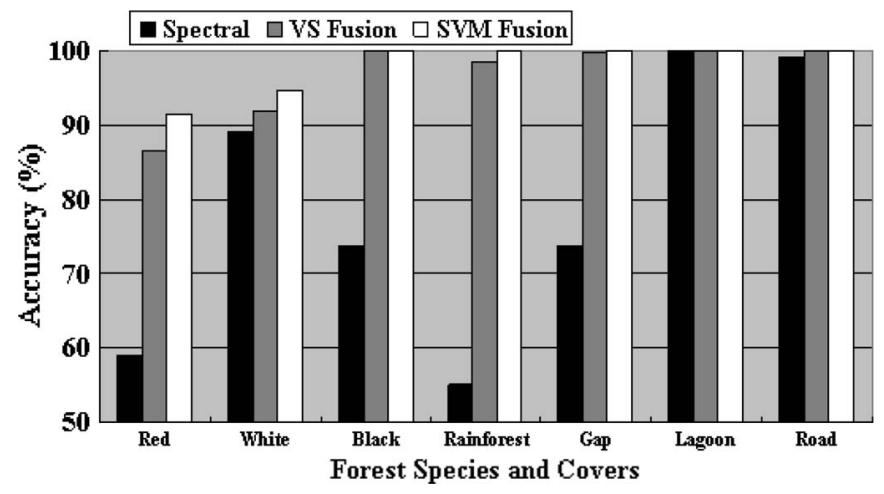

Fig. 4. Class-specific accuracies for the information fusion methods.

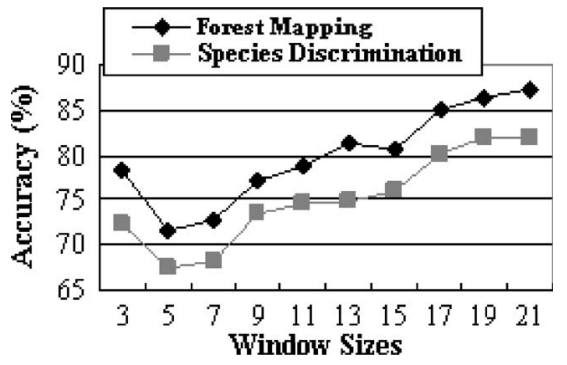

Fig. 5. Results of the second-order GLCM texture.

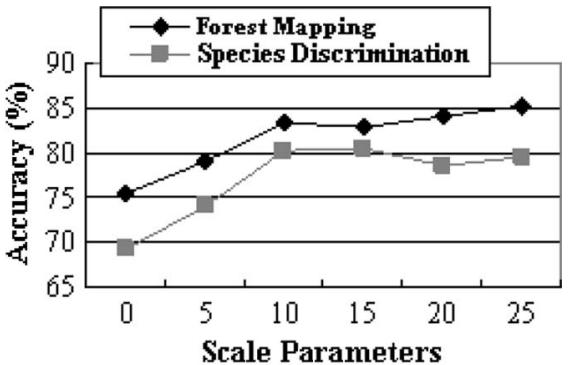

(a)

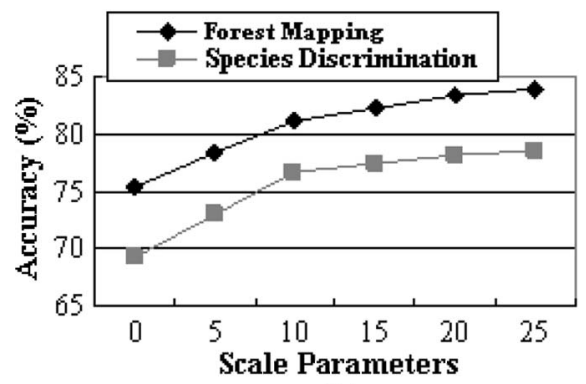

(b)

Fig. 6. Results of (a) the object-based and (b) the multilevel object-based analyses with different scale parameters.

the NDVI image. The first- and second-order statistics were stacked with the multispectral bands and then classified using SVM. The first-order statistic (variance) did not obtain satisfactory results, and the highest forest mapping accuracy (79.5\%) was given with a $3 \times 3$ window. The results of the second-order statistics (contrast, entropy, and correlation) are shown in Fig. 5. The $21 \times 21$ window size gave the most accurate result- $-87.2 \%$ for forest mapping and $81.9 \%$ for species discrimination. On the other hand, the results of object-based classification are shown in Fig. 6, where the horizontal axis represents the scale 


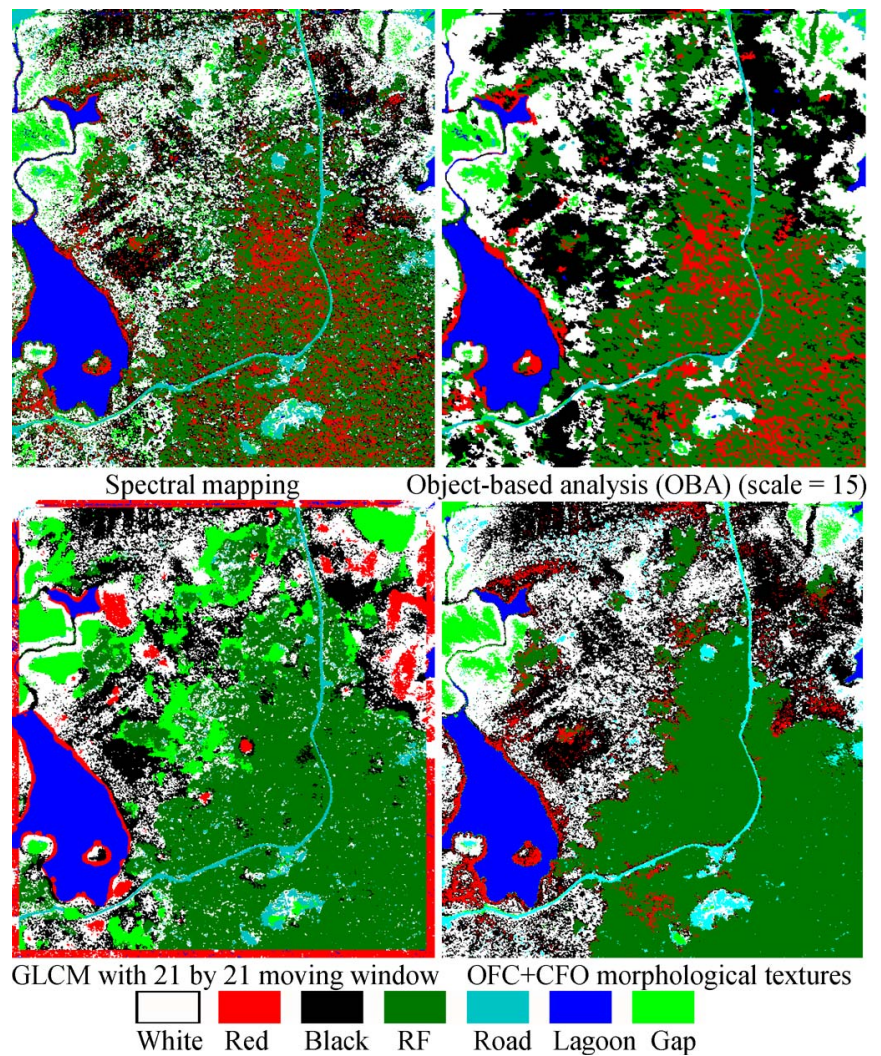

Fig. 7. Maps derived from different features.

parameters. By analyzing the results, the following could be found: 1) the optimal scales $(s)$ for forest mapping $(s=25)$ and species classification $(s=15)$ are not the same, and 2) although the multilevel approach gave higher accuracies with increasing number of levels, it did not provide additional improvements than the single-scale analysis. The classification maps for spectral features, object-based analysis, GLCM, and the $[\mathrm{OFC}+\mathrm{CFO}]$ are shown in Fig. 7.

\section{CONCLUSION}

This letter evaluated the feasibility of morphological texture features for mangrove forest mapping and species discrimination using IKONOS multispectral imagery. It is found that the morphological operators OFC and CFO can give promising results for both forest mapping (91.4\% and 93.7\%) and species classification $(89.1 \%$ and $91.1 \%$ ), which significantly outperformed the GLCM texture and the object-based analysis. The sensitivity analysis in Fig. 3 showed that the morphological textures could achieve satisfactory results in a wide parameter range and that the morphological profiles could give compa- rable results with the highest accuracies obtained by the sensitivity analysis. In addition, we developed a spectral-textural fusion model based on the function outputs of spectral and textural SVMs. The experiment revealed that, compared with the vector-stacking approach, about $2.0 \%$ improvement could be obtained by additional SVM computation.

\section{ACKNOWLEDGMENT}

The acquisition of remote sensing imagery was supported by a grant to L. Wang from the U.S. National Science Foundation (DEB-0810933). The authors would like to thank the associate editor and reviewers for their helpful suggestions which significantly improved this letter.

\section{REFERENCES}

[1] L. Wang, W. Sousa, P. Gong, and G. S. Biging, "Comparison of IKONOS and QuickBird images for mapping mangrove species on the Caribbean coast of Panama," Remote Sens. Environ., vol. 91, no. 3/4, pp. 432-440, Jun. 2004.

[2] J. Gao, "A hybrid method toward accurate mapping of mangroves in a marginal habitat from SPOT multispectral data," Int. J. Remote Sens., vol. 19 , no. 10, pp. 1887-1899, May 1998.

[3] J. Gao, "A comparative study on spatial and spectral resolutions of satellite data in mapping mangrove forests," Int. J. Remote Sens., vol. 20, no. 14 , pp. 2823-2833, Jul. 1999.

[4] E. P. Green, C. D. Clark, P. J. Mumby, A. J. Edwards, and A. C. Ellis, "Remote sensing techniques for mangrove mapping," Int. J. Remote Sens., vol. 19, no. 5, pp. 935-956, Mar. 1998.

[5] R. Bellens, S. Gautama, L. Martinez-Fonte, W. Philips, J. C. Chan, and F. Canters, "Improved classification of VHR images of urban areas using directional morphological profiles," IEEE Trans. Geosci. Remote Sens., vol. 46, no. 10, pp. 2803-2813, Oct. 2008.

[6] R. C. Gonzalez and R. E. Woods, Digital Image Processing. Englewood Cliffs, NJ: Prentice-Hall, 2002.

[7] B. Waske and J. A. Benediktsson, "Fusion of support vector machines for classification of multisensor data," IEEE Trans. Geosci. Remote Sens., vol. 45, no. 12, pp. 3858-3866, Dec. 2007.

[8] J. A. Richards and X. Jia, Remote Sensing Digital Image Analysis: An Introduction. Berlin, Germany: Springer-Verlag, 1999.

[9] G. J. Hay, T. Blaschke, D. J. Marceau, and A. Bouchard, "A comparison of three image-object methods for the multiscale analysis of landscape structure," ISPRS J. Photogramm. Remote Sens., vol. 57, no. 5, pp. $327-$ 345, Apr. 2003.

[10] L. Bruzzone and L. Carlin, "A multilevel context-based system for classification of very high spatial resolution images," IEEE Trans. Geosci. Remote Sens., vol. 44, no. 9, pp. 2587-2600, Sep. 2006.

[11] M. Pesaresi and J. A. Benediktsson, "A new approach for the morphological segmentation of high resolution satellite imagery," IEEE Trans. Geosci. Remote Sens., vol. 39, no. 2, pp. 309-320, Feb. 2001.

[12] I. Epifanio and P. Soille, "Morphological texture features for unsupervised and supervised segmentations of natural landscapes," IEEE Trans. Geosci. Remote Sens., vol. 45, no. 4, pp. 1074-1083, Apr. 2007.

[13] P. Soille and M. Pesaresi, "Advances in mathematical morphology applied to geoscience and remote sensing," IEEE Trans. Geosci. Remote Sens., vol. 40, no. 9, pp. 2042-2055, Sep. 2002.

[14] L. Wang, J. Silvan, and W. Sousa, "Neural network classification of mangrove species from multiseasonal IKONOS imagery," Photogramm. Eng. Remote Sens., vol. 74, no. 7, pp. 921-927, Jul. 2008 